\title{
Gastrointestinal Involvement in Henoch Schon- lein Purpura : A Case Report
}

Siti Kamariah $\mathrm{CM}^{\mathrm{a}}$ and Rohaizan $\mathrm{Y}^{\mathrm{b}}$

a Department of Radiology, International Islamic University Malaysia, Pahang.

${ }^{b}$ Department of Radiology, Universiti Sains Malaysia, Kelantan.

\section{ABSTRACT}

Henoch-Schonlein purpura or anaphylactoid purpura is a systemic vasculitis of unknown cause that affects small vessels and mainly involves the skin, joints, gastrointestinal tract and kidneys. Gastrointestinal involvement occurs in more than half of patients and is thought to be related to edema and intramural haemorrhage. Radiologically the gastrointestinal findings are mainly those of bowel ischemia with "thumbprinting" and bowel wall oedema. Although this disease is usually treated conservatively, aggressive intervention is occasionally performed because of acute abdominal symptoms due to complications, such as perforation, intussusception and obstruction. This report illustrates a case of Henoch-Schonlein purpura with acute abdominal symptoms due to intussusception.

KEYWORDS: Henoch-Schonlein purpura, Intussusception, Intramural haematoma

\section{CASE REPORT}

A 3-year-old Malay boy presented with one week history of multiple maculopapular rash involving both lower limbs. This was associated with fever, left knee pain and abdominal pain, which became more severe and associated with abdominal distension. Clinical examination revealed a lethargic looking child with dehydration, distended abdomen and vague mass at the right iliac fossa region associated with hyperactive bowel sounds. Ultrasound abdomen showed evidence of ileocolic intussusception (Figures 1 and 2).

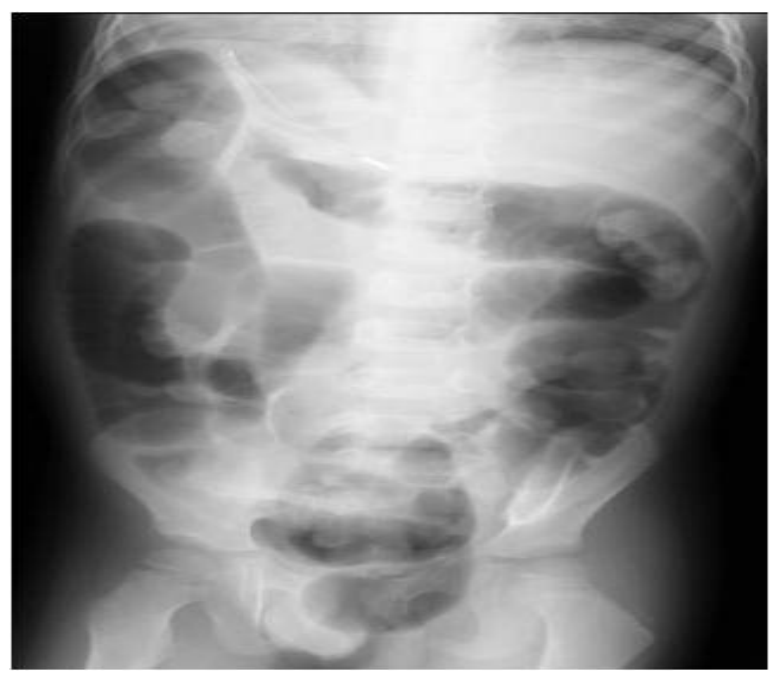

Corresponding author;

Siti Kamariah Che Mohamed, Department of Radiology, International Islamic University Malaysia, Jalan Penjara, 25100 Kuantan, Pahang Darul Makmur, Malaysia. email: skamariah08@gmail.com
Figure 1. Supine abdominal radiograph showing dilated small bowels with faecal-loaded large bowels and soft tissue opacity at the ileocaecal region.

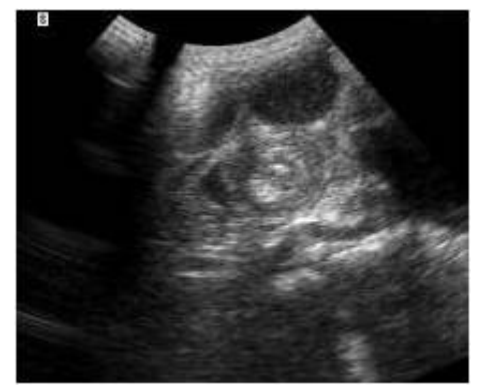

$2 \mathrm{a}$

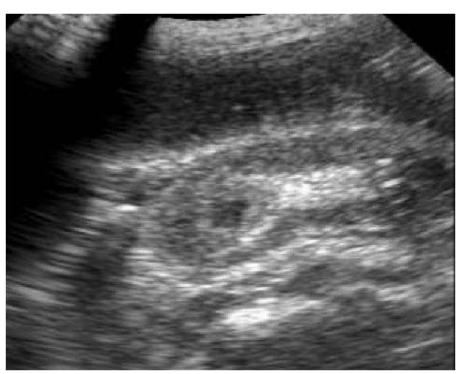

$2 \mathrm{~b}$

Figure 2. Focal bowel mass in the right iliac fossa region with 'target sign appearance' on transverse scan (2a) and 'pseudokidney sign appearance' on longitudinal scan $(2 b)$.

Air reduction intussusception was done and the patient condition improved slightly, however he still had abdominal pain with distension. Single contrast barium enema followed by CT scan abdomen was done for reassessment and to exclude recurrence of intussusception. Single contrast barium enema showed unre 
ception. Single contrast barium enema showed unremarkable findings. However, CT scan revealed short segment of oedematous small bowel at the right iliac fossa region with intramural haemorrhage, but no recurrence of intussusception (Figure 3). Following this, patient was managed conservatively and discharged well.

Figure 3: Short segment thickened oedematous small bowel at the right iliac fossa region (measures $2.5 \mathrm{~cm}$ in length) with intramural air. Hyperdensity within the oedematous bowel wall (HU of 50-60) suggests underlying haemorrhage.

\section{DISCUSSION}

Henoch-Schonlein purpura (HSP) is the most common cause of non-thrombocytopenic purpura in children and is twice more common in males than in females, and children are much more commonly affected than adults. Half of all cases occur in children under the age of 5 , and $90 \%$ of affected patients are under 10 years of age. Gastrointestinal symptoms are present in up to $75 \%$ of patients, with symptoms including colicky abdominal pain, vomiting, intestinal haemorrhage and intussusception. The natural history of HSP is self-limiting, unless complications occur. ${ }^{1}$

Intussusception is a rare gastrointestinal complication, but it is the most common surgical indication of anaphylactoid purpura as it is often ileo-ileal type (about $50 \%$ of cases) and difficult to reduce by radiologic reduction. The sonographic hallmark of intussusception has been described as the target "doughnut" or "bull's-eye" appearance on axial images and a pseudokidney structure on longitudinal images. Intussusception is usually easily diagnosed by the CT finding of a sausage-shaped mass with alternating layers of low and high attenuation. Prolonged intussusception may result in bowel necrosis and perforation. Mortality from intussusception is unusual. However, morbidity is increased by a delay in diagnosis, thus possibly creating bowel wall necrosis and perforation.

Spontaneous reduction has been reported, but surgery is advised when radiologic reduction is unsuccessful. Surgical intervention, rather than radiologic reduction is also preferable for older children suffering from anaphylactoid purpura with intussusception, where a lead point lesion is often found. As for this patient, radiologic reduction was still attempted as there were no features of perforation or peritonitis. Apart from that, ultrasound findings were also suggestive of ileocolic type of intussusception, where the success rate for radiologic reduction is usually higher.

Other radiographic findings of gastrointestinal involvement in Henoch-Schonlein syndrome are mucosal fold thickening, pseudotumours and intestinal hypomotility. Radiographic changes are those of intramural bleeding, and all these changes are reversible. Most of the studies noted about equal involvement of all parts of the small bowel, but Glasier et al, in their series found that there were predominance of duodenal and jejunal involvement. ${ }^{2}$ Intramural haematoma can serve as a lead point in a patient with Henoch-Schönlein purpura with intussusception. ${ }^{3}$ The hemorrhage is usually located in the submucosal layer of the bowel and originates from a small vessel that produces slow bleeding.

The sonographic appearance of acute intramural hematoma consists of a thickened and echogenic submucosal layer. ${ }^{3,4}$ CT scan has contributed to the early and accurate recognition of intramural haematoma. The findings include homogeneous and symmetric intramural thickening with hyperdense material $(30-80 \mathrm{H})$ in the bowel wall, which can be seen during the first 10 days after the onset of symptoms. Prompt and early recognition of intramural hematoma by the radiologist is an important contribution to patient treatment as this may prevent unnecessary exploratory surgery. In conclusion, Henoch-Schonlein purpura is a common condition, but can present with complications that need urgent intervention and aggressive treatment in order to reduce the morbidity and mortality.

\section{REFERENCES}

1. Macedo TA, Weenig RH. Henoch-Schonlein purpura. Appl Radiology Online 2003; 32(11)

2. Glasier CM, Siegel MJ, McAlister WH, Shackelford GD. Henoch-Schonlein syndrome in children: Gastrointestinal manifestations. Am J Roentgenol 1981; 136:1081-5

3. Maher AB, Joseph MC, Kevin WO. Spontaneous intramural small-bowel hematoma: Imaging findings and outcome. Am J Roentgenol 2002; 179: 1389-94

4. Frisoli JK, Desser TS, Jeffrey RB. Thickened submucosal layer: A sonographic sign of acute gastrointestinal abnormality representing submucosal edema or haemorrhage. Am J Roentgenol 2000; 175:1595-9 